\title{
Brain Tumor Segmentation on MRI Brain Images with Fuzzy Clustering and GVF Snake Model
}

\author{
A. Rajendran, R. Dhanasekaran
}

\author{
Arthanari Rajendran \\ Professor and HoD, \\ Department of Electronics and Communication Engineering, \\ Sriguru Institute of Technology, \\ Coimbatore,Tamilnadu, India \\ E-mail:rajendranav@gmail.com \\ Raghavan Dhanasekaran \\ Professor, Director-Research, \\ Syed Ammal Engineering College, \\ Ramanathapuram, Tamilnadu, India \\ E-mail:rdhanashekar@yahoo.com
}

\begin{abstract}
Deformable or snake models are extensively used for medical image segmentation, particularly to locate tumor boundaries in brain tumor MRI images. Problems associated with initialization and poor convergence to boundary concavities, however, has limited their usefulness. As result of that they tend to be attracted towards wrong image features. In this paper, we propose a method that combine region based fuzzy clustering called Enhanced Possibilistic Fuzzy C-Means (EPFCM) and Gradient vector flow (GVF) snake model for segmenting tumor region on MRI images. Region based fuzzy clustering is used for initial segmentation of tumor then result of this is used to provide initial contour for GVF snake model, which then determines the final contour for exact tumor boundary for final segmentation. The evaluation result with tumor MRI images shows that our method is more accurate and robust for brain tumor segmentation.
\end{abstract}

Keywords: Deformable model; FCM; Segmentation; MRI image; GVF

\section{Introduction}

The accurate and automatic segmentation of brain tumor on MRI image is of great interest for assessing tumor growth and treatment responses, enhancing computer-assisted surgery, planning radiation therapy, and constructing tumor growth models. This is very difficult task in existing methods. The existing methods are divided into region-based and contour-based methods. Region-based methods [1-9] seek out clusters of pixels that share some measure of similarity. These methods reduce operator interaction by automating some aspects of applying the low level operations, such as threshold selection, histogram analysis, classification, etc. They can be supervised or non-supervised. In general these methods take advantage of only local information for each pixel and do not include shape and boundary information. Contour-based methods [10-14] rely on the evolution of a curve, based on internal forces and external forces, such as image gradient, to delineate the boundary of brain structure or pathology. These methods can also be supervised or non-supervised. In general these methods suffer from the problem of determining the initial contour and leakage in imprecise edges.

In this paper we propose a method that is a combination of region-based fuzzy clustering method called Enhanced Possibilistic Fuzzy C-Means (EPFCM) and Gradient vector flow (GVF) snake model to remove the problems using the capabilities of each one. For example a region-based method can solve the problem of the initialization of a contour-based method (GVF snake model) and a contour-based method is able to improve the quality of region-based segmentation at the boundary of objects. 
So the proposed method has two main phases for tumor segmentation on MRI brain images namely, initial segmentation which is done by a region-based method and final segmentation that is performed by a boundary-based GVF snake model. We discuss these approaches in the following section.

\section{Region-Based Enhanced Possibilistic Fuzzy C-Means (EPFCM)}

In this proposed Enhanced Possibilistic Fuzzy C-Means (EPFCM) method, distance metric $D_{i j}$ in PFCM [15] is modified in such a way that it includes membership, typicality and both local, nonlocal spatial neighbourhood information to overcome the noise effect in MRI brain medical images. This modified distance metric is incorporated into objective function of PFCM. Then resultant algorithm is called Enhanced Possibilistic Fuzzy C-means (EPFCM) is obtained for enhanced segmentation results. Therefore objective function of our proposed EPFCM is defined as follows,

$$
J_{m}(U, V, T ; X)=\sum_{i=1}^{c} \sum_{j=1}^{n}\left(a \mu_{i j}^{m}+b t_{i j}^{\eta}\right) D_{i j}^{2}+\sum_{i=1}^{c} \gamma_{i} \sum_{j=1}^{n}\left(1-t_{i j}\right)^{\eta}
$$

Where, the modified distance metric is given by

$$
\begin{gathered}
D^{2}\left(x_{j}, v_{i}\right) \&=\& D_{i j}^{2}=\left(1-\lambda_{j}\right) d_{l}^{2}\left(x_{j}, v_{i}\right)+\lambda_{j} d_{n l}^{2}\left(x_{j}, v_{i}\right) \\
\sum_{i=1}^{c} \mu_{i j} \&=\& 1 \forall j, 0 \leq \mu_{i j}, t_{i j} \leq 1 \text { and } a>0, b>0, m>1, \eta>1
\end{gathered}
$$

The membership function:

$$
\mu_{i j}=\left[\sum_{i=1}^{c}\left(\frac{D_{i j}}{D_{k j}}\right)^{\frac{2}{m-1}}\right]^{-1}
$$

Typicality:

$$
t_{i j}=\frac{1}{1+\left(\frac{b}{\gamma_{i}} D_{i j}^{2}\right)^{\frac{1}{\eta-1}}}
$$

Cluster centre:

$$
v_{i}=\frac{\sum_{j=1}^{n}\left(a \mu_{i j}^{m}+b t_{i j}^{\eta}\right) x_{j}}{\sum_{j=1}^{n}\left(a \mu_{i j}^{m}+b t_{i j}^{\eta}\right)}
$$

In the following equation is suggested to compute $\gamma_{i}$ :

$$
\gamma_{i}=\frac{K \sum_{j=1}^{n} \mu_{i j}^{m} D_{i j}^{2}}{\sum_{j=1}^{n} \mu_{i j}^{m}}, K>1
$$

\subsection{Importance of modified distance metric term $\left(D_{i j}\right)$}

The modified distance metric or dissimilarity measure is rewritten from Equation (2) as follows,

$$
D^{2}\left(x_{j}, v_{i}\right) \&=\& D_{i j}^{2}=\left(1-\lambda_{j}\right) d_{l}^{2}\left(x_{j}, v_{i}\right)+\lambda_{j} d_{n l}^{2}\left(x_{j}, v_{i}\right)
$$


Where, $d_{l}$ is the distance metric influenced by local spatial information. This added local spatial neighborhood term is similar to the one which is used in [16] to incorporate the neighborhood effects in the classic FCM.The local spatial constraint is evaluated by the feature difference between neighboring pixels in the image.

$d_{n l}$ is the distance measurement influenced by non- local spatial information. This added non local term is obtained from the non local means (NL-means) algorithm [17] for image denoising. The nonlocal constraint determined by all points whose neighborhood configurations look like the neighborhood of the pixel of interest. $\lambda_{j}$ is the weighting factor controlling the tradeoff between local and nonlocal spatial information. It varies from zero to one.

\subsection{Importance of local distance metric $\left(D_{l}\right)$}

Let $N_{j}$ denote a chosen local neighborhood configuration of fixed size with respect to a center pixel $x_{j}$. If the value of a pixel $x_{k}$ in $N_{j}$ is close to the center pixel, then $x_{j}$ should be influenced greatly by it, otherwise, its influence to $x_{j}$ should be small. According to the above description, the distance measurement influenced by local information $d_{l}$ is given by,

$$
d_{l}^{2}\left(x_{j}, v_{i}\right)=\frac{\sum_{x_{k} \in N_{j}} \omega_{l}\left(x_{k}, x_{j}\right) d^{2}\left(x_{k}, v_{i}\right)}{\sum_{x_{k} \in N_{j}} \omega_{l}\left(x_{k}, x_{j}\right)}
$$

where $d^{2}\left(x_{k}, v_{i}\right)=\left\|x_{k}-v_{i}\right\|^{2}$ is the Euclidean distance metric measure the similarity between pixel pixel $x_{k}$ and cluster centroid $v_{i}, \omega_{l}\left(x_{k}, x_{j}\right)$ is the weight of each pixel $x_{k}$ in $N_{j}$ and is given by

$$
\omega_{l}\left(x_{k}, x_{j}\right)=e^{\frac{\left|x_{k}-x_{j}\right|^{2}}{\sigma^{2}}}
$$

Where, $\sigma^{2}$ is the variance of $N_{i}$. It specifies the steepness of the sigmoid curve.

\subsection{Importance of non local distance metric $\left(D_{n l}\right)$}

The distance measurement influenced by non-local information $d_{n l}$ is computed as a weighted average of all the pixels in the image $I, x_{k} \in I$

$$
d_{n l}^{2}\left(x_{j}, v_{i}\right)=\sum_{x_{k} \in I} \omega_{n l}\left(x_{k}, x_{j}\right) d^{2}\left(x_{k}, v_{i}\right)
$$

Where the family of weight $\omega_{n l}\left(x_{k}, x_{j}\right) ; x_{k} \in I$ depends on the similarity between the pixel $x_{k}$ and $x_{j}$, and satisfies the usual conditions $0 \leq \omega_{n l}\left(x_{k}, x_{j}\right) \leq 1$ and $\sum \omega_{n l}\left(x_{k}, x_{j}\right)=1$.

The similarity between two pixels $x_{k}$ and $x_{j}$ depends on the similarity of the intensity gray level vector $v\left(N_{k}\right)$ and $v\left(N_{j}\right)$, where $N_{k}$ denotes a square neighborhood of fixed size and centered at a pixel $x_{k}$. This similarity is measured as a decreasing function of the weighted Euclidean distance $\left\|v\left(N_{k}\right)-v\left(N_{j}\right)\right\|_{2, a}^{2}$, where $a>0$ is the standard deviation of the Gaussian kernel. The pixels with a similar gray level neighborhood to $v\left(N_{j}\right)$ have larger weights in the average. These weights are defined as

$$
\omega_{n l}\left(x_{k}, x_{j}\right)=\frac{1}{Q\left(x_{j}\right)} S\left(x_{k}, x_{j}\right)
$$


Where, $S\left(x_{k}, x_{j}\right)$ is the exponential form of the similarity and $Q\left(x_{j}\right)$ is the normalizing constant. These terms are defined as,

$$
\begin{aligned}
& S\left(x_{k}, x_{j}\right)=e^{\frac{\left\|v\left(N_{k}\right)-v\left(N_{j}\right)\right\|_{2, a}^{2}}{h^{2}}} \\
& Q\left(x_{j}\right)=\sum_{x_{k} \in I} e^{\frac{\left\|v\left(N_{k}\right)-v\left(N_{j}\right)\right\|_{2, a}^{2}}{h^{2}}}
\end{aligned}
$$

The parameter $\mathrm{h}$ acts as a degree of filtering. It controls the decay of the exponential function and therefore the decay of the weights as a function of the Euclidean distance.

\subsection{Importance of trade-off parameter $(\lambda)$}

For computational purpose, the search of the similar neighborhood configuration always be restricted in a larger "search window" denoted by $\Omega_{i}$. Let $x_{j}$ be the pixel under consideration. For each pixel $x_{k}$ in the search window of size $S \times S$, calculate its exponential similarity to $x_{j}$ using Equation (13). The tradeoff parameter of $x_{j}$ is then defined as

$$
\lambda_{j}=\frac{1}{m} \sum_{i=1}^{m} S_{i}\left(x_{k}, x_{j}\right)
$$

Where $S_{i}$ represents the ith exponential similarity term in the search window and choose $m=S-1$.The parameter $\lambda_{j}$ decides the trade-off between local and non local spatial information.

\section{Algorithm for Proposed EPFCM Method}

Finally the algorithm for carrying out our proposed EPFCM for tumor segmentation of MRI brain images can now be stated from the following steps

1. Select the number of clusters,$C$, and fuzziness factor $/ m \prime$

2. Select initial class centre prototypes $v=\left\{v_{i}\right\} ; i=1,2 \cdots C$, randomly and $\in, a$ very small number

3. Select the neighbourhood size and search window size

4. Calculate modified distance measurement $D_{i j}^{2}$ using the Equation (2)

5. Update membership function $\mu_{i j}$ using $D_{i j}^{2}$

6. Update $\gamma_{i} ; i=1,2 \cdots C$, using Equation (7)

7. Update typicality using the Equation (5)

8. Update cluster centre using equation (6)

9. Repeat steps 4 to 8 until termination. The termination criterion is as follows,

$\left\|V_{t+1}-V_{t}\right\| \leq \epsilon$ where'ts is the iteration steps, $\|$.$\| is the Euclidean distance norm.$

We applied this proposed algorithm to segment tumor on MRI images. In this case, we segmented the brain image into five classes: namely, CSF (Cerebrospinal fluid), WM (White matter), GM (Gray matter), tumor and background .Due to some classification errors, there are undesired additional pixels in the tumor class. To remove these misclassified components, several binary morphological operations 
are applied to the tumor class after users defined segmentation classes are obtained (number of clusters). An opening operation is first used to disconnect the components. Then we select the largest connected component, which proved to always correspond to the tumor, even if it has a small size. Here the elementary neighborhood of the morphological operations corresponds to 6-connectivity. The result of this algorithm gives segmented tumor class as shown in Figure 1(c). This output is the initial contour for the GVF snake model.

\section{Boundary-Based GVF Snake Model}

The traditional deformable active contour model [18-19] is a curve $X(S)=[x(s), y(s)], s \in[0,1]$,that move within the image to minimize the energy function. The curve dynamically changes the shape of an initial contour in response to internal and external forces. The internal forces provide the smoothness of the contour. While the external forces push the curve move toward the desired features, such as boundaries. The object contour will be got when the energy function is minimized. The energy is defined as:

$$
E=\int_{0}^{1} \frac{1}{2}\left[\alpha\left|X^{\prime}(S)\right|^{2}+\beta\left|X^{\prime \prime}(S)\right|^{2}\right]+E_{\text {ext }}(X(S)) d s
$$

Where, $X^{\prime}(S)$ and $X^{\prime \prime}(S)$ are first and second derivatives of $X(S)$ with respect to s. The parameter $\alpha$ controls the tension of the curve and $\beta$ controls its rigidity. $E_{\text {ext }}$ is the external energy which is calculated from the image data. To minimize the energy function, the snake must satisfy the Euler equation

$$
\alpha X^{\prime \prime}(S)-\beta X^{\prime \prime \prime \prime}(S)-\nabla E_{\text {ext }}=0
$$

Then the snake is made dynamic by treating as the function of time $t$, as follows:

$$
X_{t}(S, t)=\alpha X^{\prime \prime}(S, t)-\beta X^{\prime \prime \prime \prime}(S)-\nabla E_{e x t}
$$

When the solution $X(S, t)$ stabilizes, the term $X_{t}(S, t)$ is zero. Then we get the solution of equation (18).The typical external energies include:

$$
\begin{aligned}
& E_{\text {ext }}(x, y)=-|\nabla I(x, y)|^{2} \\
& E_{\text {ext }}(x, y)=-\left|\nabla\left[G_{\sigma}(x, y) * I(x, y)\right]\right|^{2} \\
& E_{\text {ext }}(x, y)=I(x, y) \\
& E_{\text {ext }}(x, y)=G_{\sigma}(x, y) * I(x, y)
\end{aligned}
$$

Where, $G_{\sigma}(x, y)$ is a 2-D Gaussian function with standard deviation $\sigma$ and mean is zero. $\nabla$ denotes the gradient operator $*$ denotes linear convolution. These external forces have a short capture range and poor convergence to boundary concavities. To overcome these problems, Gradient vector flow snake was proposed by $X u$ and Prince [18], which uses the force balance condition as a starting point of snake. It defined a new static external force field called GVF field

$$
F_{\text {ext }}=V(x, y)=[u(x, y), v(x, y)]
$$

Where, $u$ and $v$ are the grey changes on $\mathrm{x}$-axis and y-axis of the image respectively.$F_{\text {ext }}$ can be got by minimizing the following energy function:

$$
\epsilon=\iint \mu\left(u_{x}^{2}+u_{y}^{2}+v_{x}^{2}+v_{y}^{2}\right)+|\nabla f|^{2}|v-\nabla f|^{2} d x d y
$$


Where, $u_{x}, u_{y}, v_{x}, v_{y}$ are derivative of $\mathrm{x}$-axis and y-axis respectively. $f(x, y)$ is the edge map (using Canny edge detector) which is derived from image $I(x, y) . \mu$ is a regularization parameter governing the tradeoff between the first term and the second term in the formula. It should be set according to the noise of the image. The calculus of variations and numerical implementation discussed in [18] is used to obtain the solution of equation. This deformable contour is first initialized by the tumor class output of EPFCM method, which then moves towards the final tumor boundary.

\section{Results and Discussion}

Initially tumor MRI brain image is segmented for tumor class using EPFCM method, which then initial contour for GVF snake. Then the contour attracted towards final tumor boundary by edge map derived from the image using Canny edge detector. We set parameter $h=500$, search window size is $7 \times 7$,neighborhood window size is $3 \times 3, m=2, a=5, b=3$ and $\eta=2$ for EPFCM method to have proper segmentation result. We set $\alpha$ and $\beta$ value between 0.1 to 0.2 and $\mu$ value between 0.2 to 0.3 for GVF snake model to have final tumor boundary. The application of our combined method to 10 contrasts enhanced T1-weighted images and 5 FLAIR images shows better tumor segmentation. The results of four cases are as shown in Figure 1.

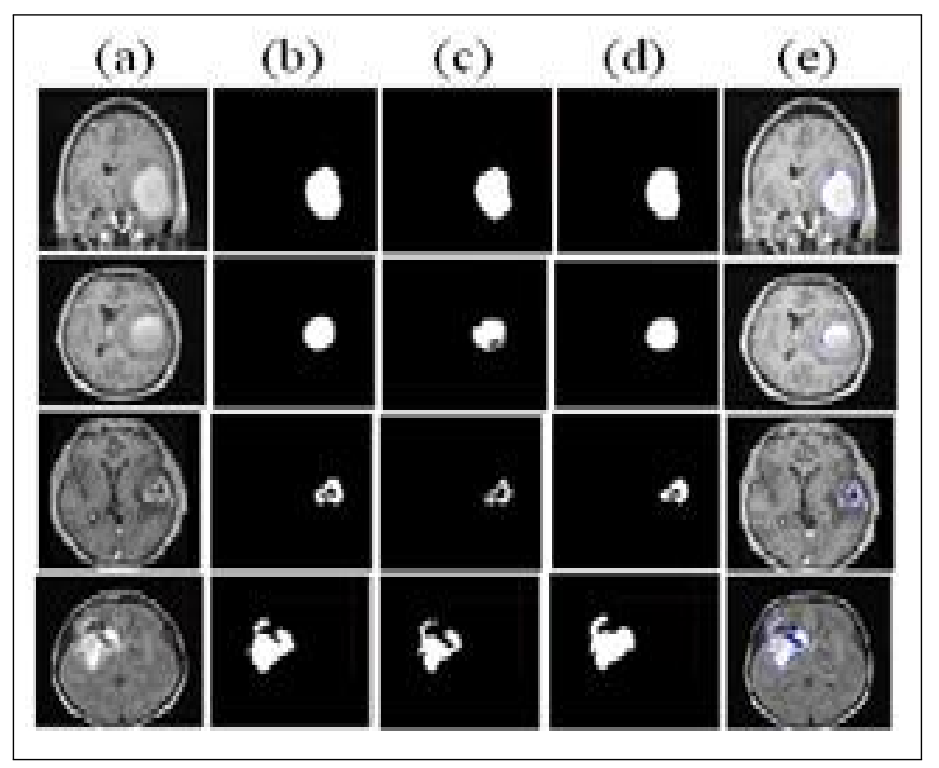

Figure 1: (a)First Column: First two images; Original CE-T1w enhanced tumors; Third image; Original CE-T1w ring enhanced tumor; Fourth image; Original non enhanced tumor FLAIR image (b) Second Column: Manual segmentation result (c) Third Column: EPFCM result showing tumor class after morphological operations (d) Fourth Column: Segmentation of tumor class using combined approach (EPFCM and GVF snake model) (e) Fifth Column: Final boundary detection (Blue curve) shows tumor region using GVF snake model.

The evaluation of segmentation performance is also carried out quantitatively by employing four volume metrics namely, the similarity index(S), false positive volume function (FPVF), false negative volume function (FNVF) and Jaccard index in our experiment. For a given image, suppose that $A_{i}$ and $B_{i}$ represent the sets of pixels belong to class $i$ in manual and in automatic segmentation, respectively. $\left|A_{i}\right|$ denotes the number of pixels in $A_{i}$. $\left|B_{i}\right|$ denotes the number of pixels in $B_{i}$.

The similarity index is an intuitive and clear index to consider the matching pixel between $A_{i}$ and $B_{i}$, 
and defined as

$$
S=\frac{2\left|A_{i} \cap B_{i}\right|}{\left|A_{i}\right|+\left|B_{i}\right|}
$$

Similarity index $S>70 \%$ indicates an excellent similarity [20].

The false positive volume function (FPVF) represents the error due to the misclassification in class $i$ and the false negative volume function (FNVF) represents the error due to the loss of desired pixels of class $i$, they are defined as follows,

$$
\begin{aligned}
\text { FPVF } & =\frac{\left|B_{i}\right|-\left|A_{i} \cap B_{i}\right|}{\left|A_{i}\right|} \\
\text { FNVF } & =\frac{\left|A_{i}\right|-\left|A_{i} \cap B_{i}\right|}{\left|A_{i}\right|}
\end{aligned}
$$

Higher value of $S$, and lower value of FPVF, FNVF gives better segmentation result.

The Jaccard index between two volumes is represented as follows,

$$
J_{i}(A, M)=\frac{\left|A_{i} \cap B_{i}\right|}{\left|A_{i} \cup B_{i}\right|} \times 100
$$

Table 1: Evaluation of the segmentation results of enhanced tumors and nonenhanced tumor by combined approach (EPFCM and GVF model) on a few CE-T1w and FLAIR images.(FET denotes the Full enhanced tumor, RET the ring-enhanced tumor,NET the enhanced tumor

\begin{tabular}{|c|c|c|c|c|c|}
\hline MRI modality type & Type of tumor & \multicolumn{4}{|c|}{ Volume metric functions (\%) } \\
\hline CE-T1w \& FLAIR & FET, RET \& NET & S & FPVF & FNVF & J \\
\hline CE-T1w & FET1 & 98.8 & 0.4 & 0.2 & 88.2 \\
\hline CE-T1w & FET2 & 96.3 & 0.7 & 0.4 & 84.5 \\
\hline CE-T1w & FET3 & 92.6 & 1.2 & 0.7 & 86.7 \\
\hline CE-T1w & FET4 & 95.7 & 0.6 & 0.6 & 89.5 \\
\hline CE-T1w & FET5 & 93.2 & 0.4 & 0.5 & 87.8 \\
\hline CE-T1w & RET1 & 95.8 & 0.1 & 0.2 & 80.2 \\
\hline CE-T1w & RET2 & 92.3 & 1.3 & 0.7 & 78.6 \\
\hline CE-T1w & RET3 & 97.6 & 0.2 & 0.3 & 76.2 \\
\hline CE-T1w & RET4 & 91.8 & 2 & 1.2 & 75.5 \\
\hline CE-T1w & RET5 & 96.3 & 0.1 & 0.3 & 77.3 \\
\hline FLAIR & NET1 & 98.8 & 0.9 & 0.6 & 76.9 \\
\hline FLAIR & NET2 & 91.5 & 2.1 & 1.8 & 83.2 \\
\hline FLAIR & NET3 & 98.6 & 2.9 & 3 & 80.1 \\
\hline FLAIR & NET4 & 94.4 & 1.8 & 2.2 & 85.2 \\
\hline FLAIR & NET5 & 95.3 & 1.2 & 2.1 & 81.6 \\
\hline \multicolumn{2}{|c|}{ Average } & 95.3 & 1.06 & 0.98 & 82.1 \\
\hline
\end{tabular}

The result of four volume metrics for our method applied to 15 tumor cases is as shown in Table 1and plotted in Figure 2. From this table, we can see that an average similarity metrics and Jaccard index of our method is $95.3 \%$ and $82.1 \%$ that is, the overlap degree between our segmentation result and the manual segmentation is higher. The average FPVF and FNVF values are equal to $1.06 \%$ and $0.98 \%$. It shows misclassification and loss of desired tumor pixels are reduced in great degree. These average values are obtained from 15 tumor cases as shown in Figure 3. To compare the results with other methods, there is no a good standard, however in comparison with works such as in [1,2,7] shows that our method has a better tumor segmentation performance. 


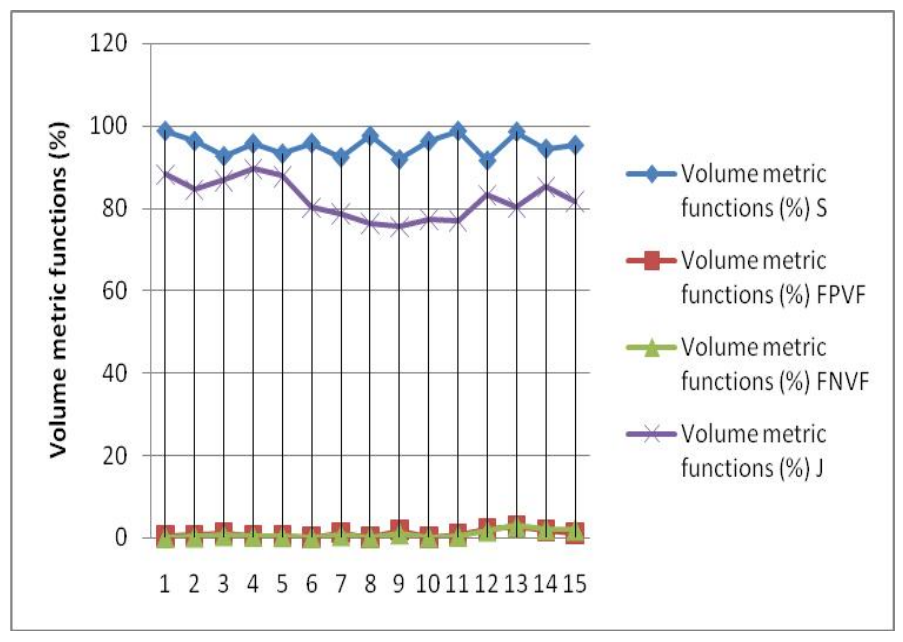

Figure 2: Graph of the quantitative comparison results of three volume metrics for 15 MRI brain tumor images.

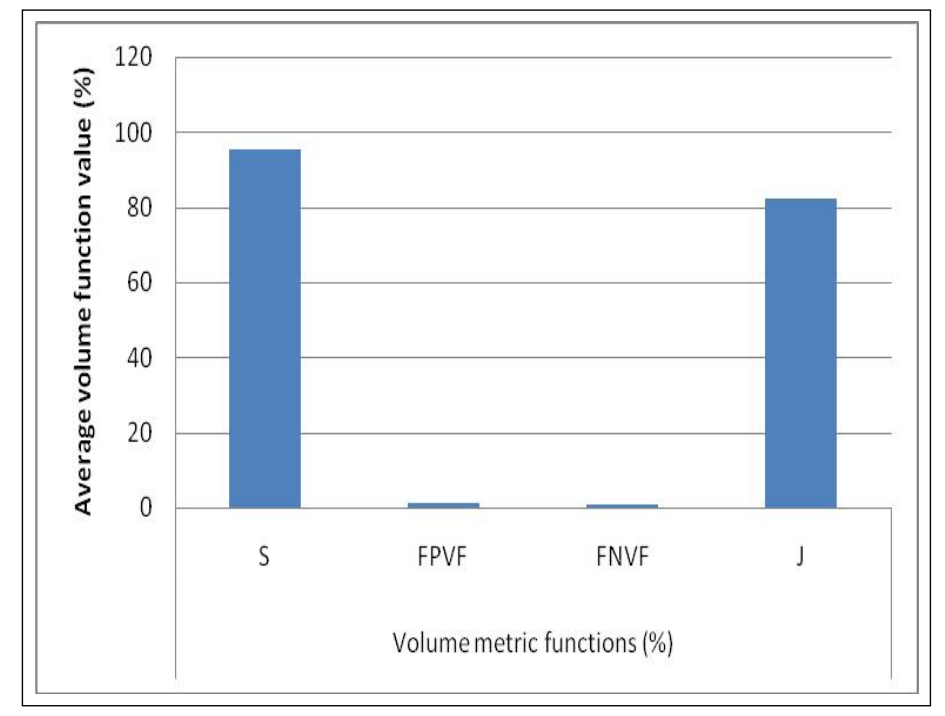

Figure 3: Graph of the average value of the three volume metrics obtained from 15 MRI brain tumor images.

\section{Conclusions}

We have presented in this paper a tumor segmentation method which combines both region based fuzzy clustering method called EPFCM and boundary based method called GVF snake model .We verified our method with brain tumour MRI images. The obtained results are quantitatively verified with other existing methods and they show that our combined approach provides better result. 


\section{Bibliography}

[1] M. Prastawa, E. Bullitt, S. Ho, G. Gerig, A brain tumor segmentation framework based on outlier detection, Medical Image Analysis, 2004, 18 (3), 217-231.

[2] J.J. Corso, E. Sharon, A. Yuille, Multilevel segmentation and integrated Bayesian model classification with an application to brain tumor segmentation, in: MICCAI2006, Copenhagen, Denmark, Lecture Notes in Computer Science, October 2006, Vol. 4191, Springer, Berlin, pp. 790-798.

[3] M.B. Cuadra, C. Pollo, A. Bardera, O. Cuisenaire, J. Villemure, J.-P. Thiran, Atlas-based segmentation of pathological MR brain images using a model of lesion growth, IEEE Transactions on Medical Imaging, 2004,23 (10), 1301-1313.

[4] J.-P. Thirion, Image matching as a diffusion process: an analogy with Maxwells demons, Medical Image Analysis, 1998,2 (3) ,243-260.

[5] G. Moonis, J. Liu, J.K. Udupa, D.B. Hackney, Estimation of tumor volume with fuzzyconnectedness segmentation of MR images, American Journal of Neuroradiology, 2002,23 ,352363.

[6] A.S. Capelle, O. Colot, C. Fernandez-Maloigne, Evidential segmentation scheme of multi-echo MR images for the detection of brain tumors using neighborhood information, Information Fusion, $2004,5,203-216$.

[7] W. Dou, S. Ruan, Y. Chen, D. Bloyet, J.M. Constans, A framework of fuzzy information fusion for segmentation of brain tumor tissues on MR images, Image and Vision Computing, 2007, 25 ,164-171.

[8] M. Schmidt, I. Levner, R. Greiner, A. Murtha, A. Bistritz, Segmenting brain tumors using alignment-based features, in: IEEE Internat. Conf. on Machine learning and Applications, 2005, pp. 215-220.

[9] J. Zhou, K.L. Chan, V.F.H Chong, S.M. Krishnan, Extraction of brain tumor fromMR images using one-class support vector machine, in: IEEE Conf. on Engineering in Medicine and Biology, 2005, pp. 6411-6414.

[10] A. Lefohn, J. Cates, R. Whitaker, Interactive, GPU-based level sets for 3D brain tumor segmentation, Technical Report, University of Utah, April 2003.

[11] Y. Zhu, H. Yang, Computerized tumor boundary detection using a Hopfield neural network, IEEE Transactions on Medical Imaging, 1997,16 (1) ,55-67.

[12] S. Ho, E. Bullitt, G. Gerig, Level set evolution with region competition: automatic 3D segmentation of brain tumors, in: ICPR, Quebec, August 2002, pp. 532-535.

[13] K. Xie, J. Yang, Z.G. Zhang, Y.M. Zhu, Semi-automated brain tumor and edema segmentation using MRI, European Journal of Radiology, 2005, 56 ,12-19.

[14] Wang Guoqiang, Wang Dongxue, Segmentation of Brain MRI Image with GVF Snake, Model,in: 2010 First International Conference on Pervasive Computing, Signal Processing and Applications,2010,pp.711-714.

[15] Pal, N. R., Pal, K., Keller, J. M., and Bezdek, J. C.A, Possibilistic fuzzy c-means clustering algorithm, IEEE Transactions on Fuzzy Systems, 2005, 13(4), pp.517-530. 
[16] Buades A,Coll B,Morel J-M. "A non-local algorithm for image denoising",In CVPR 2005:60-5.

[17] Ma, L. and Staunton, R. C., A modified fuzzy c-means image segmentation algorithm for use with uneven illumination patterns, Pattern Recognition, 2007, 40(11), pp.3005-3011.

[18] C. Xu and J.L. Prince, Snakes, shapes, and gradient vector flow, IEEE Trans. on Image Processing, March 1998,vol. 7, pp. 359-369.

[19] Bingrong Wu, Me Xie, Guo Li, Jingjing Gao, Medical Image Segmentation Based on GVF Snake Model IEEE Conference on Second International Intelligent Computation Technology and Automation (ICICTA 09), IEEE Press, 2009, vol. 1,Oct., pp. 637 - 640.

[20] Zijdenbos, A. P., Dawant, B. M., Margolin, R. A., and Palmer, A. C. Morphometric analysis of white matter lesions in MR images: Method and validation, IEEE Transactions on Medical Imaging, 1994;13(4):716-724. 